\title{
A NEW MAIN CONTROL ROOM for the AGS COMPLEX*
}

\author{
P. F. INGRASSIA, R. M. ZAHARATOS and O. H. DYLING \\ BROOKHAVEN NATIONAL LABORATORY \\ UPTON, NEW YORK 11973
}

\section{INTRODUCTION}

A new Main Control Room (MCR) has been built to control the accelerators of the AGS Complex. A new physical environment was produced to better control light, sound, temperature, and traffic. New control consoles were built around the work-stations that make up the distributed control system. Equipment placement within consoles and console placement within the room reflect attention to the "human factors" needs of the operator.

\section{MASTER PLAN}

The Master Plan called for a doubling of MCR floor space to 1600 sq. ft, a new lab for the accelerator operators, new office space, an MCR conference room, a relocated kitchen, and a small room for use as a distribution point for control wire and signal cable. Modification of the facility began in 1987. In order to minimize the impact on the Physics program work was carried out during subsequent summer AGS shutdowns and was completed in 1989. Further, the plan would correct many of the problems found in the 26 year old MCR: insufficient number of control stations, inadequate and poor control over lighting, inadequate heating and ventilation, high ambient noise levels, and years of accumulated control and signal wire.

Five control consoles were built and installed to increase the number of machine physicists, operators, and development personnel that could be supported simultaneously. The consoles were all designed and implemented as general purpose consoles so that a user would be able to control any facet of the accelerator operation from any of the control consoles. The consoles were arrayed in circular fashion, see Figure 1, in order to better allow the operations staff to be aware of who the users of the controls were at any time.

"Worked performed under the auspices of the U. S. Dept. of Energy

* Plant Engineering Deparment, Broolhaven National Laboratory

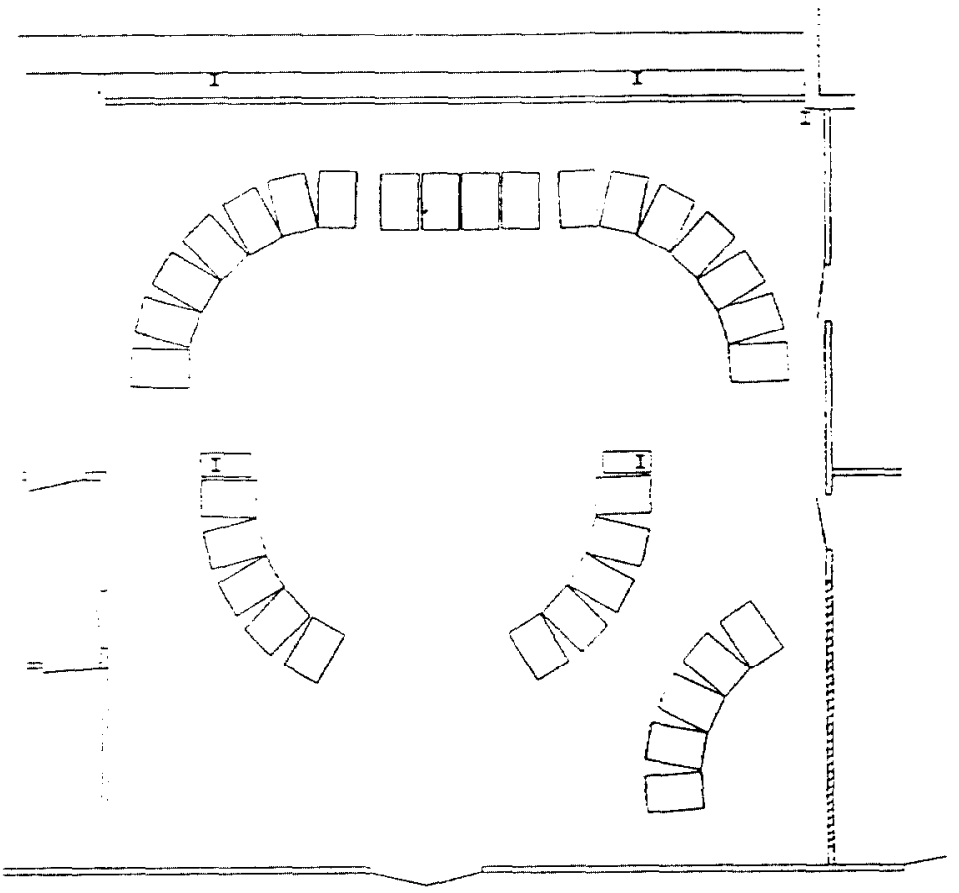

Figure 1

Lighting for the new MCR is provided by four independent concentric rings of fluorescent lighting fixtures. The outermost ring is a "wall wash" designed to provide sufficient ambient lighting for most tasks in the room and yet minimize glare on the work-station screens. The innermost rings consist of two foot by two foot fixtures that make use of anti-glare diffuses. Each console is also provided with locally controlled task lighting in the form of two incandescent spotlights aimed at the console work area.

Two air conditioning zones are provided for cooling and ventilation. The volume above the dropped ceiling is used as a supply plenum in order to provide air with reduced 
foot supply diffuses are regularly interspersed amongst the lighting fixtures. The air retum is a rom long duct located along the MCR north wall and it is an integral part of the forty foot long storage closet.

Ambient noise is controlled by a fabric covered acoustic wall tile and by acoustic ceiling tiles. Particularly noisy equipment was removed from the MCR entirely.

Hundreds of pounds of cable and wire were removed from the MCR. Cable distribution is accomplished by pathways under a new raised computer floor. A "terminal room annex ", adjacent to the north west wall of the MCR, is the source for all signal and control wire as opposed to the holes in the ceiling that previously directly fed the consoles. Signal distribution, in the terminal room annex, is done. on a per console basis and one has the ability to cross-connect signal wires between any consoles simply by installing jumper cables at this master matrix.

\section{THE CONSOLES}

Each general purpose console, see Figure 2, is made up of a core of five racks, four of which contain identical components, placed in the same location at each control station. The fifth rack contains special equipment, for example high speed oscilloscopes and each rack houses a laser printer. The heart of each control station is a 19" color monitor driven by an Apollo 4500 processor and 15" color monitor driven by an Apollo 3000 processor. The former work-station is used to run multiple graphics displays while the latter runs just two programs; one for equipment control and the other to provide switching for the analog and video signal multi-plexers [1] and oscilloscope triggers.

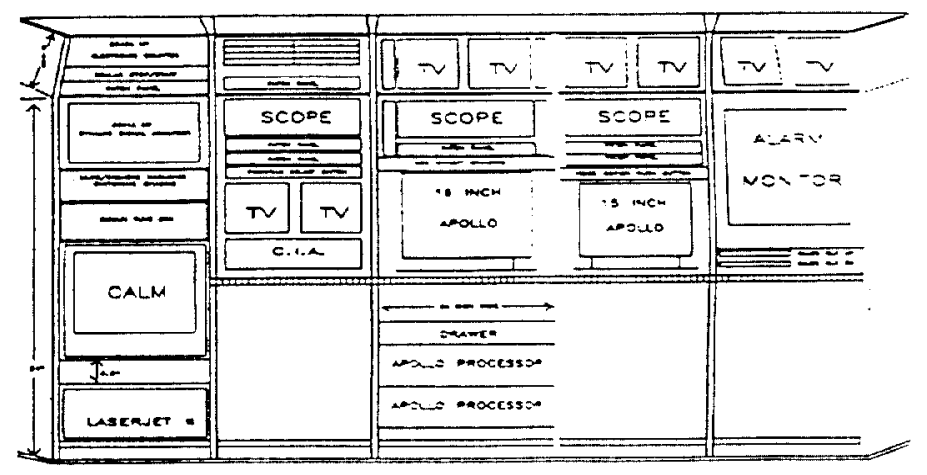

Each console supports three, four channel oscilloscopes of varying speed and capability. Eight, 9" video monitors at each console can be connected to any of 64 input videos. Each console can also reserve the input to a central video copier for hard-copy records of any video input. An alarm screen is also provided at each station.

Communications hardware was also addressed. A commercially available digital phone system, was installed to incorporate public address, two radio channels, four interphone channels, and normal telephone functions. Two such units are placed at each console.

Each console is ideally suited to support two operators. Equipment is arrayed such that the most frequently used functions are located at the center of the console. Less frequently used systems are placed at the extremes. The console wraps around the operator in two planes so that minimal head movement is required to see any of the displays.

\section{CONCLUSION}

After a year of use and some fine tuning the users are, in general, pleased with the results. The project is the result of the hard work and dedication of the MCR operations staff and a host of others in the AGS Department. Special recognition goes to Dr. Th. Sluyters and Mr. J. Grisoli whose vision and suppor made the new MCR possible.

\section{REFERENCE}

1. T. D'Ottavio, et al., "Programs for Control of an Analogue-Signal Switching Nerwork", Nuclear Instruments and Methods in Physics Research, 1990, North-Holland.

Figure 2 